\title{
Antibacterial properties of human amnion and chorion in vitro
}

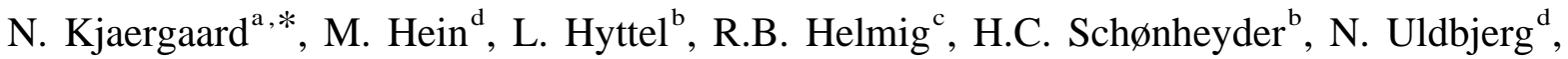 \\ H. Madsen ${ }^{\mathrm{a}}$ \\ ${ }^{a}$ Department of Obstetrics and Gynecology, Aalborg Hospital, DK-9000 Aalborg, Denmark \\ ${ }^{\mathrm{b}}$ Department of Clinical Microbiology, Aalborg Hospital, DK-9000 Aalborg, Denmark \\ ${ }^{\mathrm{c}}$ Department of Obstetrics and Gynecology, Randers Hospital, Randers, Denmark \\ ${ }^{\mathrm{d} D e p a r t m e n t ~ o f ~ O b s t e t r i c s ~ a n d ~ G y n e c o l o g y, ~ S k e j b y ~ U n i v e r s i t y ~ H o s p i t a l, ~ A a r h u s, ~ D e n m a r k ~}$
}

Received 10 May 1999; accepted 28 March 2000

\begin{abstract}
Objective: The purpose of the present study was to explore the direct effects of amnion and chorion on bacterial growth in vitro including the antibacterial spectrum. Chorioamniotic membranes were obtained under sterile conditions from 13 healthy women undergoing elective cesarean section at term. Likewise, chorioamniotic membranes were obtained from 10 healthy women with spontaneous vaginal delivery at term. Five strains of Hemolytic streptococci group B (GBS) were tested and one clinical isolate of the following species or bacterial groups: Hemolytic streptococcus group A, Staphylococcus aureus, Staphylococcus saprophyticus, Enterococcus faecalis, Escherichia coli, Pseudomonas aeruginosa, Acinetobacter calcoaceticus and Lactobacillus species. Bacteriological media included (1) blood-agar medium; (2) a transparent agar medium for submerged cultures; and (3) a nutrient broth medium. Results: An inhibitory effect of fetal membranes against a range of bacteria was found. Consistent results were obtained in experiments with cultures on agar and cultures suspended in agar (membranes from eight women in both studies). In experiments with liquid cultures (seven women) only chorion showed a marginal inhibitory effect. All strains were inhibited, but the most pronounced inhibition was obtained for streptococcus group A, S. aureus and S. saprophyticus by both chorion and amnion. Conclusion: This study demonstrated an inhibitory effect of the fetal membranes on a diverse panel of bacteria (c) 2001 Elsevier Science Ireland Ltd. All rights reserved.
\end{abstract}

Keywords: Human amnion and chorion; Hemolytic streptococci group B; Fetal membranes

\section{Introduction}

The etiology of preterm labour is multifactorial, but there is increasing evidence of infection as a cause, and especially vaginal colonization by a number of different microorganisms is associated with preterm delivery $[1,2]$. The majority of pregnancies continue until 37-42 weeks' gestation even when potentially virulent bacteria are present in the vagina. This probably indicates that competent local defence mechanisms protect the feto-maternal

\footnotetext{
*Corresponding author. Department of Obstetrics and Gynecology, Frederikshavn Hospital, DK-9900 Frederikshavn, Denmark. Tel.: +4599-202-122; fax: +45-98-422-739.

E-mail address: n.kjaergaard@dadlnet.dk (N. Kjaergaard).
}

unit and a number of studies have implicated factors such as immunoglobulins and cytokines [3,4].

Antibacterial properties are well described for amniotic fluid [5], and a limited number of studies have pointed to inhibitory effects of fetal membranes [6-8].

A rapid decline in group B streptococcal viability was observed when group B streptococci (GBS) were added to fetal membranes in an experimental model developed for studying bacterial penetration in vitro [9]. It is intriguing that, for decades, chorioamniotic membranes have been applied therapeutically to ulcerated skin surfaces, peritoneum and the lacerated eye $[10,11]$. In recent years, studies have focused on the repairing ability of amnion cells in reconstructions after microsurgery and keratectomy $[12,13]$.

The purpose of the present study was to further explore 
the direct effects of amnion and chorion on bacterial growth in vitro, including the antibacterial spectrum. Five strains of GBS were tested and one clinical isolate of the following species or bacterial groups: group A streptococcus, S. aureus, S. saprophyticus, E. faecalis, E. coli, P. aeruginosa, A. calcoaceticus and Lactobacillus species.

\section{Materials and methods}

\subsection{Fetal membranes}

Freshly delivered chorioamniotic membranes were obtained under sterile conditions from 13 healthy women undergoing elective cesarean section at term. The indications for the operations were: cephalopelvic disproportion (six women), two or more previous cesarean sections (three women), anxious for delivery (three women), previous operation for cerebral aneurysm (one woman). The membranes were intact at the time of the operation.

Likewise, chorioamniotic membranes were obtained from 10 healthy women with spontaneous vaginal delivery at term. None had been treated with antibiotics during the pregnancy.

The membranes were removed en-bloc from the placenta and cleaned in sterile, physiological saline to remove blood and debris. Amnion and chorion were easily separated by blunt finger dissection. Identification of membranes covering the cervix was not possible. Discs of amnion or chorion measuring $2-4 \mathrm{~cm}$ in diameter were used for individual experiments. The membranes were placed on the bacterial cultures immediately after birth (within $45 \mathrm{~min}$ ).

\subsection{Microorganisms}

Five strains of GBS and one clinical isolate of the following species or bacterial groups: group A streptococcus, S. aureus, S. saprophyticus, E. faecalis, E. coli, P. aeruginosa, A. calcoaceticus and Lactobacillus species.

The origins of the GBS were as follows. Three were clinical isolates: one from a neonate who died of an early-onset infection, one from a neonate without signs of serious infection, but with intrapartum signs of chorioamnionitis and one from a cervical swab from a spontaneous abortion in week 7 , respectively. The remaining two strains were obtained from M. Kilian, University of Aarhus, and in the study by Hauge et al. [14] they were included as the high-virulence clonal type 52 and low-virulence clonal type 4 , respectively.

\subsection{Media}

Bacteriological media were obtained from Statens Serum Institut, Copenhagen. Plate media included 5\% horse blood agar and transparent nutrient broth agar.
Bacterial cultures suspended into agar were prepared by adding a bacterial suspension $\left(-10^{9}\right.$ per $\left.\mathrm{ml}\right)$ to melted nutrient broth agar maintained at $50^{\circ} \mathrm{C}(1: 100)$, thoroughly mixed and then immediately poured into $9-\mathrm{cm}$ petri dishes (depth $5 \mathrm{~mm}$ ). For liquid cultures a nutrient broth medium was used.

\subsection{Bacterial cultures}

Bacterial suspensions were prepared in isotonic sodium chloride. Inocula $\left(-10^{9}\right.$ per $\left.\mathrm{ml}\right)$ were harvested from blood agar plates after incubation overnight. All plates were incubated at $35^{\circ} \mathrm{C}$ in $5 \% \mathrm{CO}_{2}$ (Steri-Cult 200, FormaScientific, Marietta, $\mathrm{OH}, \mathrm{US})$.

Cultures on agar: horse blood and nutrient agar plates were inoculated in order to obtain confluent growth.

Cultures suspended in agar: with the chosen inoculum, the transparent nutrient agar layer was made 'hazy' by the 'submerged' bacterial growth during $24 \mathrm{~h}$ of incubation.

Liquid cultures: $10 \mathrm{ml}$ of broth medium with $-10^{8}$ bacteria per $\mathrm{ml}$ were distributed into wells of polystyrene trays (no. 430343, Corning Glass Works, Corning, NY). The wells were coated with human albumin $50 \mathrm{mg} / \mathrm{ml}$ (Statens Serum Institut, Copenhagen) before use in order to block bacterial adsorption.

\subsection{Inhibition of bacterial growth}

Horse blood and nutrient agar plates: discs of either chorion or amnion were placed on the agar surface partly overlaying a cellulose nitrate filter (pore size, 0.45 or 0.2 $\mu \mathrm{m})$, (Sartorius, Göttingen, Germany). Discs and filters were removed after $24 \mathrm{~h}$ of incubation. Plates were inspected by a binocular plate microscope. The filter served as control for the occlusive effect of the fetal membrane. The partial overlay was chosen in order to determine whether an inhibitory activity, if any, was dependent on direct, cellular contact between fetal membranes and the agar surface. After reincubation for a further $24 \mathrm{~h}$, plates were inspected again in order to reveal whether growth had occurred in inhibition zones.

\subsection{Broth cultures}

A circular piece of either amnion or chorion measuring 9-10 $\mathrm{cm}^{2}$ was placed with $10 \mathrm{ml}$ of inoculated broth medium. The inoculum was initially standardized to $-10^{9}$ colony forming units $/ \mathrm{ml}(\mathrm{cfu} / \mathrm{ml})$, and $1 \mathrm{ml}$ was added to $9 \mathrm{ml}$ of the broth medium. A well without fetal membrane served as control. The polystyrene trays were placed on a gyratory shaker (M52 Minishaker, IKA ${ }^{7}$ Works, Wilmington, $\mathrm{NC}$ ) in a $35^{\circ} \mathrm{C}$ incubator in ambient atmosphere (Termaks B8000, TERMAKS ${ }^{\text {Tm }}$, Lien, Norway). After 0, 4, 8 and $24 \mathrm{~h}$ of incubation, samples of $0.5 \mathrm{ml}$ of broth medium from each well were serially diluted 1:10 in saline and aliquots of $0.5 \mathrm{ml}$ were plated on $5 \%$ horse blood agar. 
After incubation overnight, the numbers of cfus were counted manually. Saline and four different nutrient broth media were tested, and an ox-based medium was selected for the experiments.

\section{Ethics}

The project was approved by the regional research ethics committee. Informed written and verbal consent was obtained from each patient.

\section{Results}

An inhibitory effect of fetal membranes against a range of bacteria was found. Consistent results were obtained in experiments with cultures on agar and cultures suspended in agar (in both studies membranes from eight women). Table 1 shows the results of the study with fetal membranes placed on transparent nutrient broth. The results with membranes placed with cultures on horse blood agar were identical.

In experiments with liquid cultures (seven women) only chorion showed a doubtful inhibitory effect.

\subsection{Cultures on agar}

\subsubsection{GBS}

A distinct, but narrow, zone of inhibition $(1 \mathrm{~mm})$ was obtained around the edge of amnion and chorion discs. The agar plates with different GBS strains were inspected by a binocular plate microscope, and all showed a narrow 1-mm inhibition zone. No strain variation was found when GBS were studied.

Inhibition of growth was found beneath fetal membranes after incubation for $24 \mathrm{~h}$. After removal of the fetal membranes and re-incubation for a further $24 \mathrm{~h}$ no reversal of inhibition was observed. All strains of GBS were inhibited to a similar extent. The inhibition was also evident when a nitrocellulose filter was interposed between the fetal membranes and the agar surface. When GBS were overlaid by the filter alone semiconfluent growth of GBS was found.

\subsubsection{Other bacterial strains}

All strains belonging to other genera were inhibited in the same way as GBS. Inhibition was obtained with $S$. saprophyticus and although $P$. aeruginosa grew profusely beyond the membrane discs, no evidence of regrowth after removal of the membrane discs occurred.

\subsection{Cultures suspended in agar}

\subsection{1. $G B S$}

The same inhibition as described with culture on agar was found. The inhibition was pronounced beneath the fetal membranes. The most powerful inhibition was seen in the agar at the edge of the fetal membranes.

\subsubsection{Other bacterial strains}

Again, all the strains were inhibited by fetal membranes, but the inhibition was more pronounced for some of the microorganisms than for others, i.e., convincing inhibition of $S$. aureus, S. saprophyticus and group A streptococci.

At the area where the filter was partly overlaid by amnion or chorion a distinctive inhibition was found beneath the fetal membranes, i.e., the filter did not prevent the inhibition.

No growth was found in the inhibition zones after removal of the fetal membranes and re-incubation for a further $24 \mathrm{~h}$. The inhibition was more pronounced at the border of the amnion and chorion, where the specimen had been cut out (Figs. 1 and 2). When swabs were taken from the agar surface where the fetal membranes had been laying no positive culture could be found.

\subsubsection{Study with broth cultures}

Experiments showed that the density of $S$. saprophyticus and GBS was within $1 \log _{10}$ of the $-10^{8} \mathrm{cfu} / \mathrm{ml}$ found in

Table 1

Results of study with eight fetal membranes placed on transparent nutrient broth ${ }^{\mathrm{a}}$

\begin{tabular}{llll}
\hline Microorganisms & $\begin{array}{l}\text { Fetal membranes } \\
\text { (eight membranes) }\end{array}$ & $\begin{array}{l}\text { Fetal membrane } \\
\text { alone }\end{array}$ & $\begin{array}{l}\text { Fetal membrane } \\
\text { overlaying a filter }\end{array}$ \\
\hline $\begin{array}{l}\text { Group B streptococcus } \\
\text { (five strains) }^{\mathrm{b}}\end{array}$ & Amnion & $31^{\mathrm{d}}, 9^{\mathrm{e}}$ & $31^{\mathrm{d}}, 9^{\mathrm{e}}$ \\
Other species or & Chorion & $31^{\mathrm{d}}, 9^{\mathrm{e}}$ & $31^{\mathrm{d}}, 9^{\mathrm{e}}$ \\
bacterial groups $^{\mathrm{c}}$ & Amnion & $7^{\mathrm{d}}, 1^{\mathrm{e}}$ & $7^{\mathrm{d}}, 1^{\mathrm{e}}$ \\
\hline
\end{tabular}

${ }^{a}$ The inhibition of bacterial growth with and without a micro-pore filter (Sartorius) interposed between the fetal membranes and the agar was recorded. Five strains of GBS and one clinical isolate of the other species or bacterial groups were tested. The inhibition zone was found unchanged after 1-5 days, i.e., no bacteria was seen or could be cultured from the inhibition zones.

${ }^{\mathrm{b}}$ Five strains of GBS were tested.

${ }^{\mathrm{c}}$ Group A streptococcus, E. faecalis, S. aureus, S. saprophyticus, Lactobacillus, E. coli, P. aeruginosa and Acinetobacter.

${ }^{\mathrm{d}}$ No colonies were found underneath the membranes.

${ }^{\mathrm{e}}$ Few colonies were found scattered in the agar. 

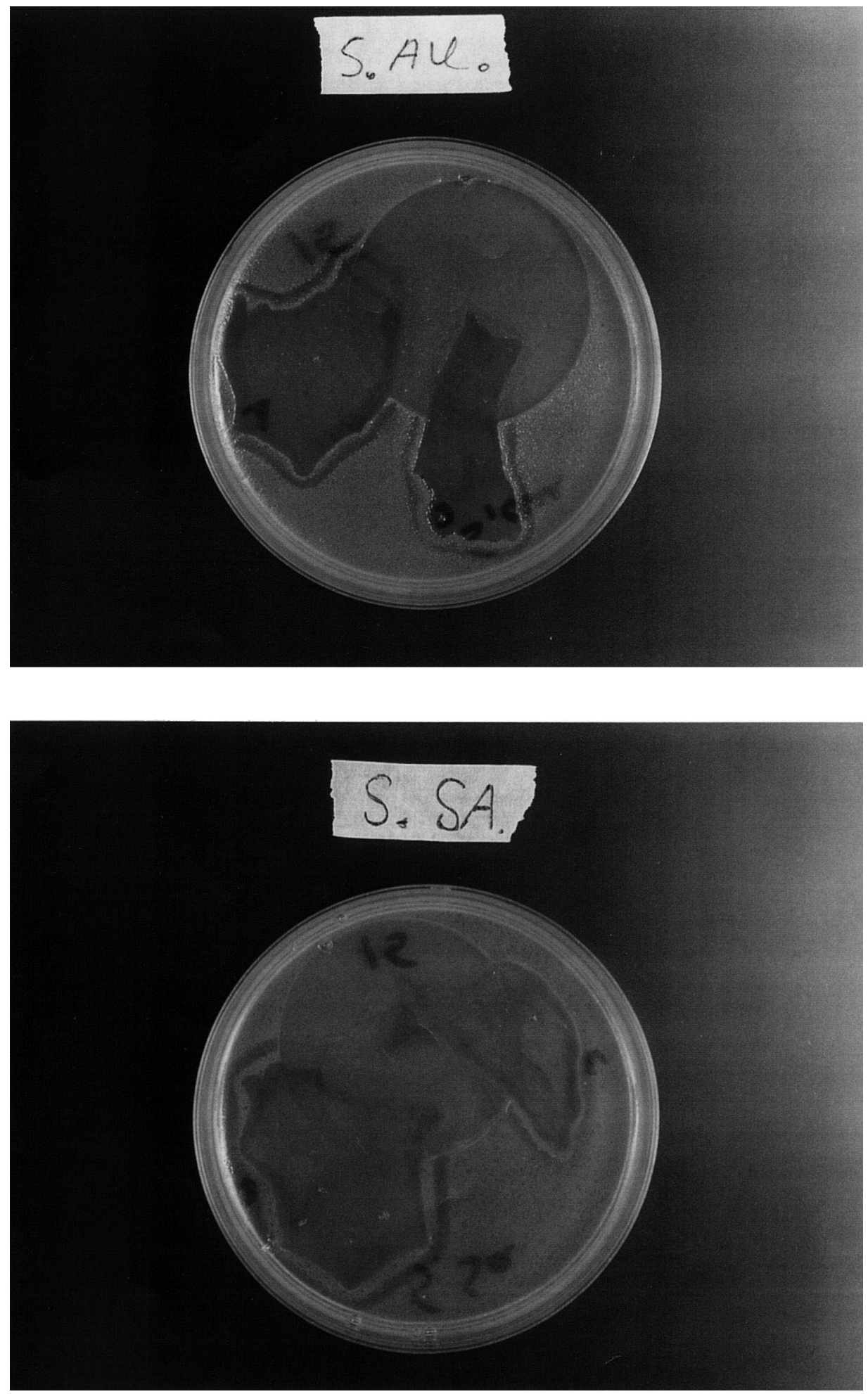

Fig. 1. S. aureus (s.au) and S. saprophyticus (s.sa) 'submerged' in ox-based medium. On both plates amnion and chorion have been partly overlaying a circular filter and the impression in the agar is obvious. Beneath the amnion and chorion, the agar is transparent as an expression of inhibition of growth of the bacteria. This is also found where the amnion and chorion have been overlaying the filter. At the place where only the filter has been lying, the agar layer is 'hazy' from bacterial growth. 


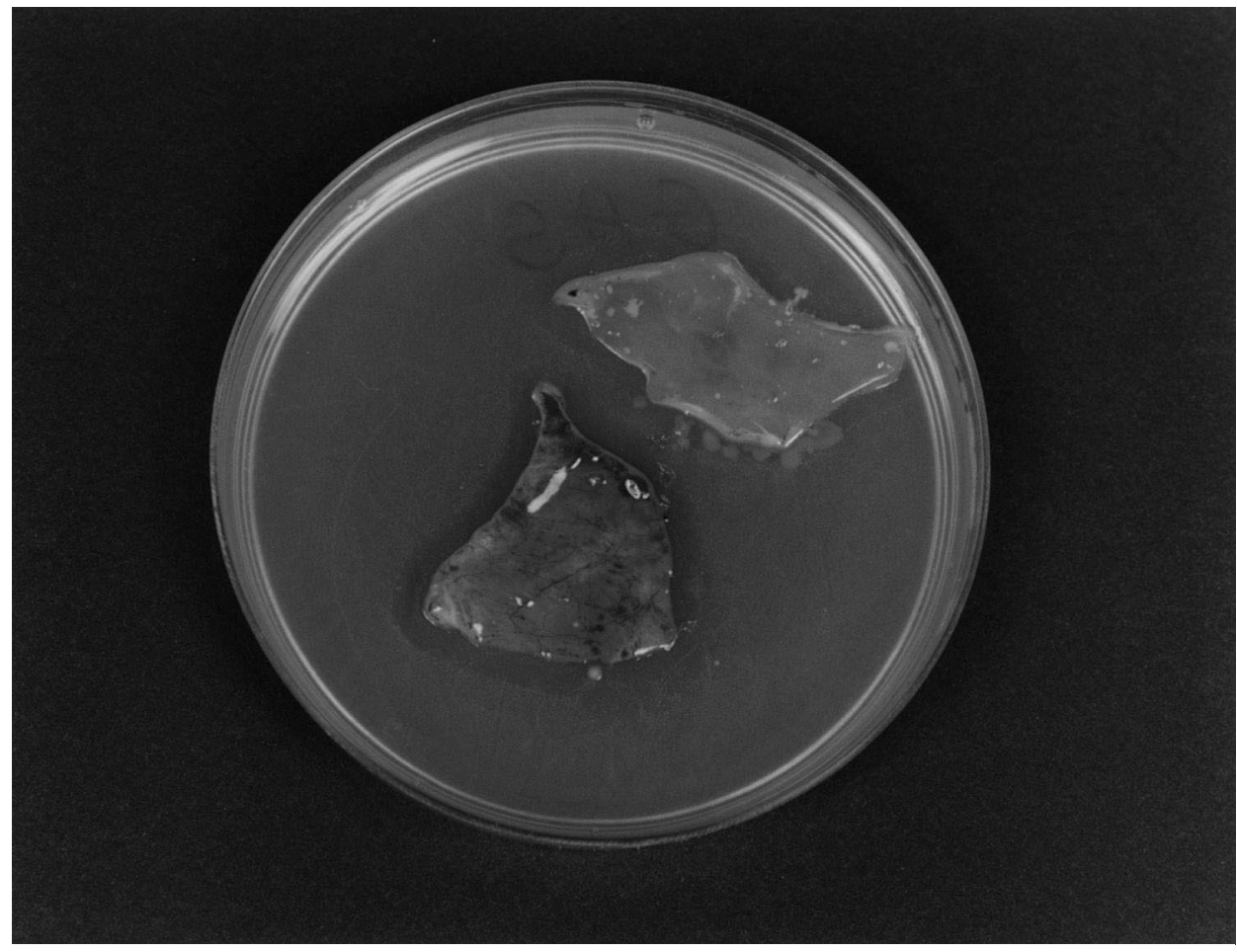

Fig. 2. Group A streptococcus 'submerged' in ox-based medium with amnion and chorion. A distinct but narrow zone of inhibition (3 mm) was obtained around the edge of the amnion and chorion.

the reservoirs without fetal membranes. Thus, chorion showed only a marginal inhibition of bacterial growth, whereas none was observed with amnion.

\section{Discussion}

This study demonstrated an inhibitory effect of the fetal membranes on a diverse panel of bacteria. The experiments with plates showed a consistent inhibitory effect by amnion and chorion, but in liquid culture only a marginal inhibitory effect by chorion was demonstrated. For the liquid culture study, we made an effort to determine the optimal nutrient broth medium, in order to avoid spontaneous decline of GBS count. It is important to use an appropriate broth culture medium [15].

The inhibition was more pronounced for some of the microorganisms than for others. Group A streptococcus, $S$. aureus and S. saprophyticus showed a pronounced inhibition by both chorion and amnion. A convincing clear zone of inhibition was seen beneath and especially at the edge of amnion and chorion (Figs. 1 and 2).

IgA has been demonstrated in the chorioamniotic membranes near the rupture edge [16]. We would suggest that the inhibition demonstrated in this in vitro experiment is produced by some other factor, because no inflammatory response could be expected in these in vitro experiments with fetal membranes from normal pregnancies at term.

Antibacterial properties of amniotic fluid are well docu- mented. It has been demonstrated that amniotic fluid contains lysozymes, 7S immunoglobulin, $\beta_{1 \mathrm{c}} / \beta_{1 \mathrm{a}}$ globulin and $\operatorname{IgA}[5,16,17]$.

Whether these are retained within the fetal membranes is uncertain. In this study the membranes were washed carefully in physiological saline before use to avoid amniotic fluid on the surface of the membranes.

Talmi et al. [18] has demonstrated an inhibitory effect of amniotic membranes, chorioamniotic membranes and synthetic polyurethane-based membranes when placed on agar plates seeded with bacteria. The intimate adherence of the membranes to the surface of the agar and the microorganisms was suggested to be the dominant factor in this inhibitory action.

We studied (1) microorganisms submerged in agar and (2) inhibition when a filter was interposed between the fetal membranes and the agar plates. In these two situations no direct contact between microorganisms and fetal membranes were obtained, but an inhibition was found.

The effect on microorganisms seems to be bactericidal and can be demonstrated in the two in vitro models presented. When swabs were taken from the agar surface where the fetal membranes had been laying positive culture was not found.

It is probably a tissue factor capable of passing a filter that exerts the effect. Direct contact between tissue and microorganisms is not necessary. Contamination of the fetal membranes was not observed, and this could indicate an independent antimicrobial purification. 
Peptides with antimicrobial activity, referred to as defensins, are synthesized by a number of epithelial cells, and may play a protective role in the genital tract $[19,20]$. Defensins exert an inhibitory activity towards a range of microbial agents. Their exact role in relation to the fetoplacental unit remains to be elucidated.

In this study fetal membranes from healthy, normal pregnancies were studied. In advance it was known that the women had a normally developed resistance to microorganisms, i.e., there was no evidence of infection of the fetomaternal unit. Other levels of resistance to microorganisms will probably be seen in infected, pregnant women.

Further studies are needed in order to characterize and quantify the antimicrobial activity of fetal membranes. The modulation of this activity during pregnancy as well as inter-individual variation may contribute to different susceptibility for obstetric infection.

\section{Acknowledgements}

We are indebted to Ms. Marlene Hylle and Ms. Maiken Jensen for excellent technical assistance. Financial support was received from Stinne \& Martinus Sørensen's Fund, the Northern Jutland Fund for Medical Research, and Aalborg Stift's Fund.

\section{References}

[1] Lamont RF. New approaches in the management of preterm labor of infective aetiology. Br J Obst Gynaecol 1998;105:134-7.

[2] Romero R, Quintero R, Nores J et al. Amniotic fluid white blood cell count: A rapid and simple test to diagnose microbial invasion of the amniotic cavity and predict preterm delivery. Am J Obstet Gynecol 1991;165:821-30.

[3] Ismail MA, Yang S, Abusharif BS, Moawad AH. Immunoglobulins in prolonged ruptured membranes. Am $\mathrm{J}$ Obstet Gynecol 1985;153:390-3.

[4] Romero R, Gomez R, Ghezzi F, Yoon BH, Mazor M, Edwin SS,
Berry SM. A fetal systemic inflammatory response is followed by the spontaneous onset of preterm parturition. Am J Obstet Gynecol 1998;179:186-93.

[5] Thadepalli H, Bach VT, Davidson EC. Antimicrobial effect of amniotic fluid. Obstet Gynecol 1978;52:198-204.

[6] Robson MC, Krizek TJ. The effect of human amniotic membranes on the bacterial population of infected rat burns. Ann Surg 1973;177:144.

[7] Robson MC, Samburg J, Krizek TJ. Quantitative comparison of biological dressings. Surg Forum 1972;23:503-7.

[8] Burleson R, Eiseman B. Mechanism of antibacterial effect of biological dressings. Ann Surg 1972;2:181-6.

[9] Kjaergaard N, Helmig RB, Schønheyder HC, Uldbjerg N, Hansen ES, Madsen H. Chorioamniotic membranes constitute a competent barriere to group B streptococcus in vitro. Eur J Obstet Gynecol Reprod Biol 1999;83:165-9.

[10] Colocho G, Graham WP, Green AE, Matheson DW, Lynch D. Human amniotic membrane as a physiologic membrane. Arch Surg 1974;109:370-3.

[11] Trelford JD, Trelford-Sauder M. The amnion in surgery, past and present. Am J Obstet Gynecol 1979;134:833-45.

[12] Quintero RA, Carreno CA, Yelian F, Evans MI. Repair kinetics of amnion cells after microsurgical injury. Fetal Diagn Ther 1996;11:348-56.

[13] Kim JC, Tseng SC. Transplantation of preserved human amniotic membrane for surface reconstruction in severely damaged rabbit corneas. Cornea 1995;14:473-84.

[14] Hauge M, Jespersgaard C, Poulsen K, Killian M. Population structure of streptococcus agalactiae reveals an association between specific evolutionary lineages and putative virulence factors but not disease. Infect Immun 1996;64:919-25.

[15] Philipson EH, Palermino DA, Robinson A. Enhanced antenatal detection of group B streptococcus colonization. Obstet Gynecol 1995;85:437-9.

[16] Cunha SP, Berezowski AT, Costa MW, Ribeiro SR, Duarte G. Demonstration of the presence of $\operatorname{IgA}$ in the human chorioamniotic membranes. Int J Obstet Gynecol 1984;22:107-10.

[17] Schlievert P, Johnson W, Galask RP. Amniotic fluid antibacterial mechanisms: newer concepts. Semin Perinatol 1977;1:59-70.

[18] Talmi YP, Sigler L, Inge E, Finkelstein Y, Zohar Y. Antibacterial properties of human amniotic membranes. Placenta 1991;12:285-8.

[19] Zhao C, Wang I, Lehrer RI. Widespread expression of beta-defensin hBD-1 in human secretory glands and epithelial cells. FEBS Lett 1996;396:319-22.

[20] Svinarich DM, Gomez R, Romero R. Detection of human defensins in the placenta. Am J Reprod Immunol 1997;38:252-5. 